\title{
ANL/NDM-49 \\ SOME COMMENTS ON RESOLUTION AND THE ANALYSIS AND INTERPRETATION OF EXPERIMENTAL RESULTS FROM DIFFERENTIAL NEUTRON MEASUREMENTS
}

by

\author{
Donald L. Smith \\ November 1979
}

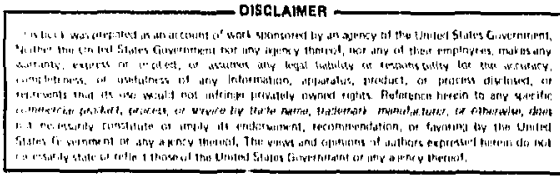

Applied Physics Division

Argonne National Laboratory

9700 South Cass Avenue

Argonne, Il11nois 60439

USA 
The Nuzlear Data and Measurements Series presents results of studies in the fiold of microscopic nuclear data. The primary objective is the dissemination of information in the comprehenst e form required for nuclear technology applications. This Series is devoted to: a) measured microscopic nuclear parameters, b) experimental techniques and facilities employed in measurements, c) the analysis, correlation and interpretation of nuclear data, and d) the evaluation of nuclear data. Contributions to this Series are reviewed to assure technical competence and, unless otherwise stated, the cortents can be formally referenced. This Series does not supplant formal journal publication but it does provide the more extensive information required for technological applications (e.g., tabulated numerical data) in a timely manner. 
ABSTRACT. ............................................

I. INTRODUCTORY REMARKS. ............................

II. DEFINITION OF TERMS AND DISCUSSTON OF CONCEPTS............. 3

III. COMPARISON OF RESULTS FROM DIFFERENT EXPERIMENTS............. 16

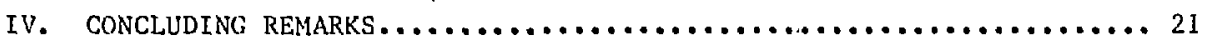

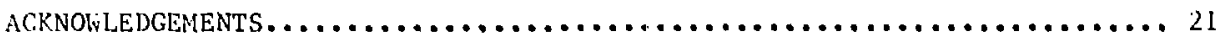

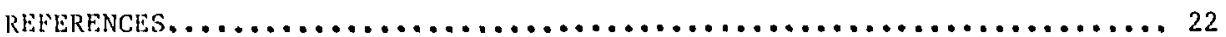

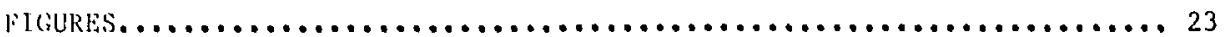


SONE CONMENTS ON RESOLUTION AND THE ANALYSIS AND INTERPRETATION OF EXPERIMISNTAL RESULTS FROM

DIFFERENTIAL NEUTRON MEASUREMENTS*

\author{
by \\ Donald L. Smith \\ Argonne National Laboratory \\ Argonne, Illinois 60439 \\ U.S.A.
}

ABSTRACT

Effects of finite resolution in differential neutron measurements are examined. General procedures for deriving the experimental resolution functions from a knowledge of the experimental parameters are presented. Problems encountered in the comparison of different data sets, when the measured cross section is known to fluctuate with energy, are discussed. This report has been prepared with the objective of encouraging closer attention to the matter of resolution by experimenters, evaluators and users of neutron nuclear data.

${ }^{*}$ This work supported by the U. S. Department of Energy. 


\section{INTRODUCTORY REMARKS}

The civelopment of various nuclear-energy technologies has resulted in. a broad range of needs and increasingly stringent accuracy requirements for nuclear data upon which these technologies are founded. For example, accuractes of $\sim 2-5 \%$ for the fast-brecder reactor dosimetry differeptial cross section data base have been established as program objectives. $\Lambda$ present, experimental techniques are not quite up to meeting that requirement and a near-term goal of $\sim 4-7 \%$ seems more realistic except for a few speciai cases. ${ }^{1}, 2$ Accuracy goals of $\sim 1-3 \%$ have been established for certain key actinide cross sections such as ${ }^{235} \mathrm{U}(\mathrm{n}, \mathrm{f}) .{ }^{3}$ This objective has not been achieved either.

Considerable attention is now being devoted to such matcers as neutr $n$ flux measuremert, standard cross sections, improved knowledge of nuclear decay schemes, conversion parameters, etc. This effort is necessary, but not. sufficient. It is now well known that nany neutron cross sections which are important for nuclear technology fluctua:e dranatically with energy and angle. For some applications, only the average behavior is of importance. For others, the implications of this structure are profound (e.g. shielding). In the past decade; improved experimental resolution has revealed signtficant structure up into the MeV-energy range, especially for medium mass elements $(\Lambda=20-70)$ which are used as coolants or in structural components of nuclearenergy devices. An important point which is often overlooked is that the existence of structure in the microscopic cross sections can serfously hampor at tempts to accurntely mensure these cross sections (e.g. Ref. 4). In cases where the cross sections exhibit a great denl of structure, differences in the results of various mensurements mity be dus in large part to structural effects rather than to difficulties in measuring neutron fluence or to inconsistencies in the use of other muclear parameters required for the experiments. Figure $l$ is an example which demonstrates the gravity of this problem. This figurc indicates the confusion which is introduced by attempting to compare the results of various measurements of a given cross section without regard to the effects of structure on the experimental process.

There are problems which may never be entirely resolved. Precise, detailed comparison of the results of measurements of fluctuating cross sections may not be possible unless experimenters acquire and report more information about their work than appears to be practical. In fact, the volume of information wich is now being generated is severely straining the procedures and institutions which have traditionally been used to exchange scientific results (reports, journals, data center files, etc.). However, it is suggested that considerable improvement beyond the present state of affairs is possible without drastic modification of current experimental and communicational practices. If the accuracies which are now being sought for the nuclear data underlying various technologies are to be achieved, it wil] be necessary for scientists to agree upon the definitions of and relationships between various experimental quantities normally used to express the results of their work. Furthermore, it will be necessary for investigators to analyze the results of their measurements in terms of the parameters of their specific experiments to a greater extent than has been the practice heretofore. This is not an unreasonable expectation since much of the requisite analytical work can be performed using digital computers. Of course, there is the additional responsibility of having to 
monitor the sensitive parameters of a measurement which must be borne by the investigator. It is of little practical value to calculate cross sections and deduce resolution functions carefully from a detalled computer model of the experiment ii, unbeknown to the experimenter, certain parameters which are assumed to be known are actually not well known initially or drift away from initial values in an undetermined manner.

Of concern here are matters which are intimately dependent upon specific experimental details. These details are often omitted from discussion when the results of experimental work are reported. This might be proper when the main objective of the paper is not to provide numerical results, but rather to confirm a hypothesis or to report upon a new physical phenomenon. However, if the objective of the work is the provision of numerical values, then experimenters have the obligation to provide sufficient information to enable a critical evaluation of their results. Regardless of whether the details are reported or not, the experimenters should take it upon themselves to pay attention to the significant details, and they ought to report their work in such a way as to convince others that this has been done. The present report addresses this area of the experimental process. Agreement on terms is important and compromise is not only a practical necessity, but is unavoldable in principle. No attempt is made to review these matters comprehensivety or to treat them in a mathematically rigorous fashion. The goal of this work is to stimulate those who deal with nuclear data to think about this aspect of their work, and to remind users who seek accuractes of better than a few percent of some of the difficulties involved in satisfying their requirements. report:

The following topics are addressed in the remaining sections of this

i) The relationship between the microscopic cross section and measured or calculated quantities of the experiment is examined. A suggested definition for the "measured" cross section is presented and its interpretation is discussed with the aid of a hypothetical experiment.

ii) The concept of resolution is explored and procedures for determining it are suggested.

iii) Procedures for correcting data for secondary neutrons are discussed.

iv) Experimental probiems encountered in measurements of broad resolution cross sections with thick samples in the presence of resonance structure are liscussed.

v) Some problems associated with the comparison of various differential data sets are mentioned. 


\section{DEFINIIION OF TERMS AND \\ DISCUSSION OF CONCEP'TS}

The cruss section $\sigma$ is an abstract quantity which is defined in terms of other parameters which can, in principle, be measured in he laboratory. The relationship between cross section and the principal experimental parameters is given (in simplest form) by

$$
\Delta \mathrm{Y}_{\mathrm{m}}=\mathrm{F} N \mathrm{~N} \Delta \mathrm{X} \sigma
$$

where

$$
\begin{aligned}
\Delta Y_{m}= & \text { observed reaction rate }\left(\mathrm{sec}^{-1}\right), \\
F= & \begin{array}{l}
\text { flux of monoenergetlc neutrons in a uniform parallel beam } \\
\left(\mathrm{cm}^{-2} \cdot \sec ^{-1}\right),
\end{array} \\
N= & \text { number of sample atoms per unit volume in a uniform sample } \\
& \left(\mathrm{cm}^{-3}\right), \\
\Lambda= & \text { sample area normal to the neutron bean }\left(\mathrm{cm}^{-2}\right), \\
\Delta \mathrm{x}= & \text { sample thickness }(\mathrm{cm}), \\
\varepsilon= & \text { rntio of the observed reacton } y \text { ield to the true reaction rate } \\
& (\text { dimengtonless efficiency factor), } \\
\sigma= & \text { cross section per atom }\left(\mathrm{cm}^{2}\right) .
\end{aligned}
$$

Equation (I) defines what is known as the microscoplc cross section. This relat lonship between the measurable quantities $\Delta \mathrm{r}_{\mathrm{m}}, \mathrm{F}, \mathrm{N}, \mathrm{A}, \Delta \mathrm{x}$ and $\varepsilon$ and the ferived $\sigma$ is valid only to the extent that the sample is sufficiently small so that a very small fraction of the incident neutrons experience interactions.

Equation (I) represents the relationship between a set of measurable quantities and the abstract microscopic cross section in an ideal experiment. Complications arise because the relationship between the measured parameters of a realistic experiment and the abstrart microscopic cross section is usually much more complicated than the one given in Eq. (1). The term "differential" applies whenever one or more parameters (energy, angle, etc.) are limited to a narrow range. However, if the cross section o fluctuates considerably within the parameter range defined by the experiment, it is difficult to compare the results from one measurement with those from another experiment having different parameter limits. Experimenters who routinely perform what are known as "integral experiments" (e.g. reactor spectrum measurements) realize the difficulty of comparing their results with other integral experiments. "Differential experiments" are actually integral experiments with somewhat tighter limits on the experimental parameters, but often not tight enough to avoid serious interpretational problems. In short, the same problems encountered in integral experiments are also encountered in many differential experiments. 
The diversity of techniques utilized in neutron cross section measurements is so great that it is difficult to discuss concepts in a general way. This report considers some of these concepts witli the aid of a hypothetical experiment which includes features of transmission experiments used to measure total cross sections, of scattering experiments involving detection of either neutrons or gamma rays and of activation measurements. Although the present discussion assumes a neutron source with characteristics typical for measurements with monoenergetic accelerators, the conceptual changes are often minor for white-source measurements where timing conditions clefine thi: energy limits. We assume that neutrons emanate from a limited region of space. The neutron energies and flux depend on direction owing to kinematic effects and characteristic properties of the source reaction. Additional energy spread is introduced by target effects (e.g. the charged particles from the accelerator lose varying amounts of energy there). Neutrons which stcike the sample are subjected to scattering and absorption. Likewise, the secondary radiation which is detected in order to observe the nuclear process (e.g. the scattered neutrons in scattering experiments) may be scattered or absorbed before reaching the detector. Detector efficiency is also dependent on factors such as geometry, energy, etc. All of these experimental. effects can be taken into consideration symbolically tn a generalization of iq. (1),

$$
y_{\mathrm{II}}=\int W(\rho) \sigma(\rho) d \rho
$$

where "o" is il vector whtch represents all the parameters of the experiment. Integration extends over the experimental range for these parameters in Eq. (2) and all subseiplent expressions. Conventionally, one defines a cross section in terms of vilutibles such as energy and angle; however, $\mathrm{Eq}$. (2) is acceptable because eargy and angle can be derived from the experimental paratueters $p$. 'lhe eipriment is differential if it is designed so as to limit the range of possible energies or angles (or both).

In a good experiment, it is possible to define the experimental parameters and to determine the measured raction rate to within uncertainties which can be estimated rather we11. However, Eq. (2) is of little use because it simply states that the observer reaction rate is related to the average of the unknown microscopic cross section over the range of experimental parameters. In order to make progress, it is necessary to define a quantity which will subsequently be called the measured cross section $\sigma_{m}$. Thus,

$$
Y_{m}=\sigma_{m} \int W(\rho) d \rho
$$

This is a more useful expression than Eq. (2). $Y_{m}$ can be measurea and $W(\rho)$ can be deduced (within uncertainties) from a knowledge of experimental details. A comparison of Eqs. (2) and (3) yields 


$$
a_{m}=\frac{\int w(\rho) \sigma(\rho) d \rho}{\int W(\rho) d \rho} .
$$

Expressed this way, the measured cross section $\sigma_{m}$ is interpreted as an average of the microscopic cross section over the parameter space of the experiment. The derived quanity $\sigma_{\mathrm{m}}$ is often the best approximation to the true cross section which can possibly be deduced from the experimental investigation.

The analysis is not conceptually complete. What is sought is the cross section as a function of energy or angle (or both). Equation (4) provides a clue as to how the average energy $F_{m}$ or angle $\theta_{m}$ should be defined to insure consistency. Thus,

$$
v_{m}=\frac{\int W(\rho) E(\rho) d \rho}{\theta_{m}=\frac{\int_{W(\rho) d \rho}}{\int W(\rho) \theta(\rho) d \rho}},
$$

It must be understood that the measured cross section $\sigma_{m}$ is not necessarily equal to the true microscopic cross section 0 corresponding to the average energy $E_{m}$ (or average angle $\theta_{m}$ in the case of an angle-cependent cross section).

Knowledge of detailed structure in the microscopic cross section is irretrievably lost by the impiied averaging of the measurement process. When the microscopic cross section varies smoothly with energy and angle, not much information is lost by averaging. If pronounced variations occur within the experimental resolution, the consequences can be serious.

The meaning of the term resolution needs to be considered. Most. commonly, it is applied to the variables energy and angle encountered in an experiment. Given the function $W$ (in terms of the experimental parameters $\rho)$, it is possible to define the normalized functions $R$ and $A$ which are projections of $W$ onto energy space or angle space in such a way that 


$$
\begin{aligned}
& E_{\mathrm{m}}=\int_{0}^{\infty} R(E) E d E, \\
& \theta_{\mathrm{n} 1}=\int_{0}^{\pi} A(\theta) \theta d \theta .
\end{aligned}
$$

In practice, the integrals are calculated for the range of energies and angles encountered in the experiment. Projections of this sort are difficult to express analytically, but can be deduced numerically with the aud of a digital computer. $R$ and $A$ are the energy resolution and angular resolution functions respecively. The procedure of projecting the weighting function $W$ onto enerny or angle space can be nisleading when the microscopic cross section varies sharply with both energy and angle within tho limits imposed by the experiment. For example, this unfortunate situation is encountered in neutron elasile scattering from medium mass nuclei. In other exporiments, it is perfectly reasonable to consider the projection of W onto a single space. For example, in activation studies only the projection onto energy space is of interest. In neutron elastic scattering studies for light elenents or vory heavy eloments, the energy dependencies are smooth and the primiry concern is with angular resolution.

It is interesting to examine some resolution functions from a specific experiment. Figure 2 shows energy resolution functions derived from the analysis of a ginma-ray experiment. Details of the experimental procedure are described in Ref. 5. liere, gamma-ray production from neutron-irradiated samples is measured with a Ge( $\mathrm{Li}$ ) detector. Both prompt and delayed (activation) measurements have been performed. The flux of neutrons produced by the ${ }^{2} H(d, n)^{3}$ He reaction was measured with a Lission detector. The shapes of the resolution functions are energy dependent. The dominant effect at low energy is energy spread introduced by passage of the deuterons through the gas target cell. The influence of kinematic effects is evident for higher energies.

The resolution functions are often not symmetric about the average energies $\mathrm{E}_{\mathrm{m}}$. The term resolution usually refers to a single parameter which in some fashion represents the "width" of the distribution. If the experimental distribution functicns were symmetric and possessed shapes which could be represented analytically using a few parameters, then the experimenter could provide a great deal of information about the experiment by merely reporting the parameters of the resolution function along with $E_{\mathrm{m}}, \sigma_{\mathrm{m}}$ (and $\theta_{\mathrm{m}}$ if applicable). In the ideal case of a Gaussion resolution fumction, a single paraneter such as the full width at half maximum would be adequate. When the resolution functions are more complicated (i.e. as in Fig. 2), the experimenter faces the dilemina of how to present the experimental results in a way which permits meaningful comparison with the 
results of other work. This is a decision which must be made by the experimenter, and the matter will not be pursued here beyond mentioning the approach selected for some of the work reported from our liaboratory. The experimental resolution is arbitrarily defined to be the full width at half maximum (FWHM) for the distribution, regardless of the shape of the distribution function. These values are reported in tabular form along with the measured cross sections. An indication of the shape trend is then provided graphically in published documentation on the experiment (e.g. see Fig. 2). When the exferimental results are inseusitive to the resolution for a particular parameter (e.g. proton emission argle for an $(n, p)$ activation measurement), then no corresponding resolution information is provided.

At this point, attention is directed to consideration of measurements that are differential in energy in order to address a problem which is encountered in many types of experiments performed at monoenergetic facjilties. Neutrons are produced by reactions such as ${ }^{2} \mathrm{H}+d+n,{ }_{\mathrm{Li}}+\mathrm{p} ; \mathrm{n}$, etc. It cannot be assumed that the primary reactions such as ${ }^{2} H(d, n)^{3} H e$, ${ }^{7} \mathrm{Li}(\mathrm{p}, n)^{7} \mathrm{Be}$, etc., are the only ones involved in the experiment. In fact, neutrons are also produced via competing reactions such as ${ }^{2} \mathrm{H}(\mathrm{d}, n \mathrm{p})^{2} \mathrm{H}$, ${ }^{7} \mathrm{Li}(\mathrm{p}, n){ }^{7} \mathrm{Be}{ }^{*},{ }^{7} \mathrm{LI}\left(\mathrm{p}, \mathrm{n}{ }^{3} \mathrm{He}\right)^{4} \mathrm{He}$, etc., when the bombarding energy is sufficiently high. The energies of these secondary neutrons are noticeably reduced relative to the primary neutrons. The formalism outlined above cannot deal with the case of a multigroup neutron sou:ce, so it must be modified. A measurement cannot be designated as properly differential in energy unless the mafor contribution to the meisured reaction rate $\mathrm{Y}_{\mathrm{m}}$ comes from a dominant primary group of neutrons with a rather well-defined energy band located higher in enersy than the sccondary neutron groups. Otherwise, the uncertainties involved in applying corrections become unacceptably large. The neutron source reactions which are most commonly used do satisfy this requirement when used over limited energy ranges.

The requisite modification of the for'nalism commences with Eq. (2). The equation

$$
y_{m}=\sum_{i} \int w(i, p) \sigma(i, p) d p
$$

replaces $\mathrm{Eq}$. (2), where the index " $i$ " identifies a specific neutron group $(i=1$ labels the dominant primary group). Some secondary reactions like ${ }^{2} \mathrm{H}(\mathrm{d}, \mathrm{np})^{2} \mathrm{H}$ and ${ }^{7} \mathrm{Li}\left(\mathrm{p}, \mathrm{n}^{3} \mathrm{He}\right)^{4} \mathrm{He}$ produce broad neutron energy spectra. To handle these, it is often necessary to break up each continuous group artifically into several subgroups or bands for the analysis. Equation (9) can be rewritten in the form

$$
\mathrm{Y}_{\mathrm{m}}=\int \mathrm{W}(1, \rho) \sigma(1, \rho) \mathrm{d} \rho+\sum_{i>1} \int W(i, \rho) \sigma(i, \rho) d \rho
$$


It is reasonable to define $\sigma_{m}$ as an average over the primary group. Then Eq. (3) can be zeneralized to the expression

$$
Y_{m}=\sigma_{m} \int W(1, \rho) d \rho+\sum_{i>1} \int W(i, \rho) \sigma(i, p) d \rho
$$

The complexity introduced by the secondary-neutron terms must be dealt with by approximation. Two approaches are nentloned below. Selaction of a methor depends on the information available and personal preferences.

One possible approach involves replacing Eq. (11) by

$$
Y_{\mathrm{n}}=\sigma_{\mathrm{n}} \int W(1, \rho) \mathrm{d} \rho+\sum_{i>1} \int W(i, \rho) \sigma_{\Lambda}(i, \rho) \mathrm{d} \rho
$$

where $\sigma_{A}$ is an approximation to the true cross section. Since the secondary neutron energtes ire lower than those of the primary group, $\sigma_{A}$ can be deduced from experiment in the following way: The experimenter commences the experiment at low energy under conditions where the secondary neutrons are either not present or are ineffective. He then proceeds toward higher encrgies. Results from the lower energy measurements are used, when required, to calculate higher-energs cross sections by means of Eq. (12). This bootstrap approach ts recommended when no information ts avallable on the cross section at the start of the experiment. llowever, it assumes that the experimenter cin span a broad range of energies. ihis is not always possible. The bootstrap approach is quite effective for threshold reactions, but is less satisfactory for renctions such as neutron capture which can exhibit large cross sections at low energy.

A second approach is possible if some prior information on the shape of the cross section over a wide range of energies is available. This may be possible if other experiments have been performed or if theoretical calculations have been made. Starting with Eq. (11),

$$
Y_{m}=\sigma_{m}\left[\int W(1, \rho) \mathrm{d} \rho+\sum_{i>1} \int W(i, \rho) \frac{\sigma(i, \rho)}{\sigma_{m}} \mathrm{~d} \rho\right]
$$

is obtained by algebraic munipulation. Then assume

$$
\frac{\sigma(i, p)}{\sigma_{m}} \approx \frac{S_{A}[E(i, p)]}{S_{A}\left(1:{ }_{m}\right)}
$$


where the function $S_{A}$ approximates the shape of the cross section over the entire range of neutron energies encountered in the experiment and $E_{m}$ is defined by

$$
E_{\mathrm{T}}=\frac{\int W(1, \rho) E(1, \rho) d \rho}{\int W(1, \rho) d \rho},
$$

analogous to $\mathrm{Eq}$. (5). The shape function $\mathrm{S}_{\mathrm{A}}$ need not be normalized, but it should be energy-averaged to the experimental resolution. Equations (13) and (14) combine to yield

$$
Y_{m}=\sigma_{m}\left\{\int W(1, \rho) d \rho+\sum_{i>1} \int W(i, \rho) \frac{S_{A}[E(i, p)]}{S_{A}\left(E_{m}\right)} d \rho\right\}
$$

Procedures for calculating $W(p)$ or $W(1, \rho)$ have not been mentioned in tise preceeding discussion. These functions depend upon the detalis of the experiment. As an example, consider the gamma-ray experiment mentioned above. The weightinf function for gamma-ray yield from the sample assumes the form

$$
\begin{gathered}
W(p)=F\left(\rho_{1}\right) \cdot A_{n}\left(\rho_{2}\right) \cdot A_{Y}\left(p_{3}\right) \cdot G\left(p_{4}\right) \\
\cdot \varepsilon\left(\rho_{5}\right) \cdot \prod_{k=6}^{n} c_{k}\left(\rho_{k}\right),
\end{gathered}
$$

where the $\rho_{k}$ are subsets of the entire parameter space for the experiment, and

$$
\begin{aligned}
& F\left(\rho_{1}\right)=\text { neutron flux factor (external to the sample), } \\
& A_{n}\left(\rho_{2}\right)=\text { neutron absorption factor, } \\
& A_{Y}\left(\rho_{3}\right)=\text { gamma-ray absorption factor, } \\
& G\left(\rho_{4}\right)=\text { geometry factor, } \\
& E\left(\rho_{5}\right)=\text { efficiency factor for the Ge(Li) detector, } \\
& C_{k}\left(\rho_{k}\right)=\text { various correction factors } \\
& \text { (e.g. neutron multiple scattering). }
\end{aligned}
$$


The presence of pronounced resonance structure in the total cross section leads to serious prcolems when attempts are made to measure total cross sections (or ar,v other cross sections) with broad resolution and thick samples. Smith and Whalen ${ }^{4}$ have shown that sizable discrepancies are observed between energy-averaged high-resolution data and the results of broad-resolution measurements of the total cross sections for medium-mass nuclei (c.s. titanium, chromium, iron, nickel, etc.). The broad-resolution results are usually observed to be systematically low relative to energyaveraged high-resolution values. The magnitude of the discrepancy increases with increased sample thickness, and it is jeverest in energy regions where high-resolution data-if available-indicate that the fluctuations are very pronounced. This effect leads to discrepancies in the determination of cross section data for applications and for the purpose of comparison with theory. It is common practice to use thick samples to improve statistical accuracy when the cross section is expected to be low-such as in a valley between resonance peaks. This will not produce a problem so iong as the resolution of the measurement is sharper than the widths of the resonance peaks and spacings between resonances. Therc is considerable evidence indicating that the widths and spacings of resonances in several mediummiss nuclei are so fine that the above-mentioned resolution criterion is not sitisfied even for recent white source measurements. 6

This problem can be exemplified by considering a hypothetical experiment in wich a tmiform slab of material with unit area and thickness $L$ is irraliated with a uniform strenm of neutrons incldent perpendicular to the surface. A cross section $\sigma_{i m}$ is determined by measuring a detector yield $\mathrm{Y}_{\mathrm{im}}$. The relationshyp between the microscople cross section and the yleld is given by the formuln

$$
Y_{i m}(L)=N \int_{a}^{\infty} d l_{i} F(E) \varepsilon(E) \sigma_{i}(E) \int_{0}^{L} d x e^{-N \sigma_{T}(E) x},
$$

where

$$
\begin{aligned}
& \mathrm{N} \quad=\text { number of sample atoms per unit valume }\left(\mathrm{cm}^{-3}\right) \text {, } \\
& F(E)=\text { incident neutron flux energy distribution external to the } \\
& \text { sample }\left(\mathrm{MeV}^{-1}\right) \text {, } \\
& E(E) \quad=\text { energy-dependent detector efficiency (no dimenston), } \\
& \sigma_{i}(E)=\text { energy-dependent microscopic cross section for the reaction } \\
& \text { processes under investigation }\left(\mathrm{cm}^{2}\right) \text {, } \\
& \sigma_{T}(F)=\text { energy-dependent mtcroscopic total cross section }\left(\mathrm{cm}^{2}\right) \text {, } \\
& \text { L = sample thickness }(\mathrm{cm}) \text {, } \\
& Y_{\text {im }}(L)=\text { detector yield with explicit dependence on sample thickness } \\
& \text { indicated. }
\end{aligned}
$$


Equation (18) is a specific example of Eq. (2). By comparison with Eq. (3), the measured cross section, also likely to be dependent upon $L$, is given by the expression

$$
\gamma_{1 m}(1,)-N a_{i m}(1,) \int_{0}^{\infty} d 1: N(1:) c(1:) \int_{0}^{1} d x e^{-N \sigma_{p}(1:) x} .
$$

Further analysis of Eq. (19) yields the expression

$$
Y_{i m}(L)=\sigma_{1 m}(L) \int_{0}^{\infty} d E F(E) \varepsilon(E) \frac{\left[1-e^{\left.-N \sigma_{T}(E) L\right]}\right.}{\sigma_{T}(E)} \text {. }
$$

Define a resolution function

$$
R(E, L)=F(E) E(E) \frac{\left\lfloor 1-e^{\left.-N \sigma_{T}(E) L\right]}\right.}{\sigma_{T}(E)} \text {, }
$$

so that Rq. (20) assumes the form

$$
Y_{i m}(L)=\sigma_{i m}(L) \int_{0}^{\infty} d E k(E, L) .
$$

By anelogy with $\mathrm{Eq} \cdot(4)$,

$$
\sigma_{i m}(L)=\frac{\int_{0}^{\infty} d E \sigma_{i}(E) R(E, L)}{\int_{0}^{\infty} d E R(E, L)} .
$$


The measured cross section is therefore a function of the sample thickness because it is an average of the microscopic cross section over a resolution functicin which is deperdent upon $L$.

Equation (20) is a perfectly reasonable way to relate the measured yield to a cross section as long as the unaveraged microscopic total cross section is used in the analysis. In other words, if a comparison were to be made between a calculated average for $\sigma_{i}$, using Eq. (18), and the measured quantity, agreement could be expected. However, a serious discrepancy could result if an average or broad-resolution total cross section is used in the analysis prescribed by Eqs. (18) or (20). What is needed is high-resolution total cross section data to properly calculate the effect of the shielding lerm,

$$
S(E, L)=\frac{\left[1-e^{\left.-N \sigma_{T}(E) L\right]}\right.}{\sigma_{T}(E)},
$$

in the $\mathrm{pr}$ isence of pronounced resonance structure. Even knowledge of the proper broad resolution cra:s section will not help to avoid this protiem.

The effects of resonanco shlelding can be understood by considering tho strightiorward spactal case where the cross section to be determined is the totial crass section. The transmisston is measured so that the yield is

$$
Y_{T N}\left(L_{1}\right)=\int_{0}^{\infty} d E R\left(E_{i}\right) E(E) e^{-N \sigma_{T}\left(B_{i}\right) L},
$$

for a sample of thickness $L$, whereas the yield is

$$
Y_{T M}(0)=\int_{0}^{\infty} d E F(E) E(E)
$$

in the absence of the sample. Therefore, the ratio of sample-in to sampleout yields corresponds to the transmission averaged over a resolution function equal to the product of $F$ and $\varepsilon$ : 


$$
\frac{Y_{T M}(L)}{Y_{T M}(0)}=\left\langle e^{-N \sigma_{T} L}\right\rangle .
$$

Ciearly,

$$
e^{-N<C>I} \neq \frac{Y_{T M}(L)}{Y_{T M}(0)}
$$

where

$$
\left\langle\sigma_{T}\right\rangle=\frac{\int_{0}^{\infty} d E F(E) E(E) \sigma_{T}(E)}{\int_{0}^{\infty} d E F(E) E(E)} .
$$

The crucial error which is commltted all ton often in the analysis of total cross section data is that Eq. (28) is treated as an absolute equality under all conditions (even for sizable $L$ ).

For very small L, Eqs. (25) and (26) combine to yleld

$$
\frac{Y_{T M}(L)}{Y_{T M}(O)}+1-N L\left\langle\sigma_{T}\right\rangle \text { for } L+0 \text {, }
$$

with $\left\langle\sigma_{\mathrm{T}}\right\rangle$ defined according to Eq. (29). For total cross section measurements with broad resolution, it is necessary to perform measurements with several sample thicknesses and then extrapolate to "zero" thickness in order to deduce broad resolution results which will agree with the average of highresolution values (when there is pronounced resonance structure). This approach often leads to problems owing to the effect of large statistical errors for small thicknesses L. 
Energy-averaged total cross sections play a useful role in theoretical analyses, but they must be used with care in applications. For example, it is apparent that erroneous results can be obtained in shielding studies.

There is a special situation for which it is possible to improve upon ones' knowledge of the cross section beyond the measured quantity $\sigma_{\pi^{*}}$ If the shape of the cross section $S(E)$ is reasonably well established from other experimental work or from theoretical arguments (i.e. information from sources beyond the experiment in question), then it is possible to unfold the effects of resolution and determine the true cross section $\sigma\left(E_{m}\right)$ at the average energy $E_{m}$.

Suppose

$$
\sigma(E)=C_{\sigma} S(E)
$$

where $C_{\sigma}$ is a normalization constant relating the shape to the true cross section. Equation (4) assumes the form

$$
\sigma_{\mathrm{m}}=\int_{0}^{\infty} R(k) \sigma(k) d t
$$

where $R(B)$ is the nornalized energy resolution function, therefore

$$
\sigma_{m}=c_{\sigma} \int_{0}^{\infty} R(E) S(E) d E .
$$

Then,

$$
C_{\sigma}=\frac{\sigma_{m}}{\int_{0}^{\infty} R(E) S(E) d E} .
$$


Consequently, from Eqs. (31) and (34),

$$
\sigma\left(E_{m}\right)=k o_{n},
$$

where

$$
k=\frac{S\left(E_{m}\right)}{\int_{0}^{\infty} R(E) S(E) d E} .
$$

So, it is seen that a knowledge of the detailed shape permits derivation of the microscupic point-wise cross section from an experimental measurement with finlte resolution. In practice, detailed shape information is available only for a few well-studied reactions where the energy dependence is smooth. New measurements are usually undertaken for these reactions only in order to refine the normalization, and the factor $k$ is aften a very small correction amounting to less than $\sim 1 \%$. The procedure described by Eqs. (31)-(36) is of little value when the cross section has pronounced structure which is not well known. 


\section{COMPARISON OF RESULTS FROM DIFFERENT EXPERIMENTS}

A practical matter which must be dealt with by experimenters, evaluators and users of nuclear data is that of comparing the results of various experiments in order to echleve improved knowledge of the fundamental cross section. This is a complex issue which cannot be dealt with readily in general terms. Unfortunately, the problem does not receive as much attention as it deserves in either experimental studies or evaluations. Only a few aspects of this problem will be addressed in this report. Energy resolution problems are probably of the greatest concern, although angular resolution effects can confuse the comparison of angular distribution measurements and thus the interpretation of the data in terms of models.

We may rewrite Eq. (32) in the form

$$
\sigma_{i, 1}=\int_{-\infty}^{\infty} R\left(\lambda-E_{m}\right) \sigma(\lambda) d \lambda
$$

whero

$$
\begin{aligned}
& \sigma_{\Pi 1} \quad=\text { mensured cross section corresponding to energy } \mathbb{E}_{m}, \\
& R\left(\lambda-\mathrm{E}_{m}\right)=\text { the normal ired energy resolution function, } \\
& \sigma(\lambda) \quad=\text { mictoscopic cross section at energy } \lambda .
\end{aligned}
$$

The cross section $\sigma$ is assuned equal to zero for negative energy in order to preserve the symmetry of the formula. ${ }^{7,8}$ There is no simple relationship between $\sigma_{7}$ and $\sigma$ if the resolution function spans an energy region where $\sigma(\lambda)$ is influetced by several resonances. Consequently, it is difficult to compare $\sigma_{m}$ deciuced from two different experiments. If $\sigma$ were known, it would be possible to determine whether a measurement of $\sigma_{\mathrm{m}}$ yielded the proper results; however, the measurement would be unnecessary if this information were available, The cross section $a$, being a physical quantity, can be represented by an analytic function and thus can be expanded into a Taylor's series. The resule is

$$
\sigma_{m}=\sigma\left(E_{m}\right)+\sum_{n=1}^{\infty} \frac{\sigma^{(n)}\left(E_{m}\right)}{n !} \int_{-\infty}^{\infty} \lambda^{n} R(\lambda) d \lambda
$$

where

$$
\sigma^{(n)}\left(E_{m}\right)=n^{\text {th }} \text { derivative of } \sigma \text { at energy } E_{m} \text {. }
$$


So, to relate $\sigma_{m}$ and $\sigma\left(E_{m}\right)$, it is necessary to consider the derivatives of $\sigma$ and moments of the distribution function. If the distribution function is symmetric,

$$
R(\lambda)=R(-\lambda),
$$

and only the even-order terms of Eq. (38) are nonvanishing.

If the cross section is smooth and free of sharp resonances within the range of Influence of the resolution function, only the lowest-order terms of Eq. (38) are significant (e.g. see Fig. $3 A)$. Then, for a symmetric resolution function,

$$
\sigma\left(E_{m}\right) \approx \sigma_{m}-\frac{1}{2} \sigma^{(2)}\left(E_{m}\right) \int_{-\infty}^{\infty} \lambda^{2} R(\lambda) d \lambda,
$$

The second derivative of the cross section can be estimated from the experimental data.. In the example from Fig. $3 \Lambda$, where the second derivative is obviously negative, the mensured cross section is smaller than the true cross section. Under these conditions, the results of two experimental meaurements could be compared rather unambiguously to each other and to the true cross section. Clearly we are dealing with essentially the same toplc as was consldered in section 1, Eqs. 31-36, although from a somewhat different point of view.

It is not possible to compare the results of two independent measurements of a fluctuating cross section in detall when both resolution functions are broader than the peak widths and spacings. However, if sufficient data are available, it may be possible to check on normalization consistency by the procedure discussed below. Furthermore, it is not possible to deduce the detailed structure of the microscopic cross section from the broad resolution results. Too much information is lost by the averaging procedures as is demonstrated in $F 1 g$. 3B.

The results of two experiments are summarized by the equations

$$
\sigma_{1}(E)=\int_{-\infty}^{\infty} R_{1}(\lambda-E) \sigma(\lambda) \mathrm{d} \lambda,
$$


and

$$
\sigma_{2}(E)=\int_{-\infty}^{\infty} R_{2}(\lambda-E) \sigma(\lambda) \mathrm{d} \lambda,
$$

where $R_{1}$ and $R_{2}$ are resolution functions for the two measurements and the average energy $E$ is presumed to be the same for both measurements. Suppose that $R_{2}$ corresponds to a higher resolving power than $R_{1}$ so that the "widths" $W_{1}$ and $W_{2}$ for these distributions satisfy the relationship

$$
w_{2}<w_{1}
$$

Equation (41) can be written in the form of a double integral,

$$
\sigma_{1}(k)=\int_{-\infty}^{\infty} \int_{-\infty}^{\infty} R_{1}\left(\lambda-k_{1}\right) \delta\left(\lambda^{\prime}-\lambda\right) \sigma\left(\lambda^{\prime}\right) d \lambda^{\prime} d \lambda,
$$

by using the Dirac delta function, $S$. It is possible to express $\sigma_{l}(E)$ in terms of: the resolution function $\mathrm{R}_{2}$,

$$
\sigma_{1}(E) \equiv \int_{-\infty}^{\infty} \int_{-\infty}^{\infty} R_{3}(\lambda-E) R_{2}\left(\lambda^{\prime}-\lambda\right) \sigma\left(\lambda^{\prime}\right) \mathrm{d} \lambda^{\prime} \mathrm{d} \lambda,
$$

through the use of a third function $R_{3}$. The interpretation of Eqs. (44) and (45) $j \equiv:$ given two complete sets of data, the higher resolution results couid. in principle, be compared to the poorer resolution results by further averaging the higher resolution results using the function $R_{3}$. In practice, there are serious difficulties involved in this approach. One concern is that, in general, $R_{3}$ is not defined uniquely. When $R_{1}$ and $R_{2}$ are Gaussian functions, then Eq. (45) is satisfied if $R_{3}$ is a Gaussian with width $W_{3}$ given by the equation

$$
w_{3}^{2}=w_{1}^{2}-w_{2}^{2} .
$$


If the resolving power of the second experiment is much better than the first experiment so that

$$
W_{2} \ll W_{1},
$$

then, From Eqs, (44), (45) and (46), the limits

$$
\begin{aligned}
& W_{3} \rightarrow W_{1}, \\
& R_{3} \rightarrow R_{1},
\end{aligned}
$$

are approached. It then becomes meaningful to compare the results of the two experiments by averaging the high resolution results using the resolution function $R_{l}$ corresponding to the poorer resolution data.

There are some important qualifications to this averaging procedure. First, the higher resolution excitation function must have been measured continuously in steps no greacer than the "width" $W_{2}$ of the resolution function $R_{2}$ over the cuthre range of euergles of concern for the comparison. The purpose for this is to insure that any structure wileh inght be present: is sultibly delineaced or avernged, as the case may be, by the oxperiment. If the cross section is "smooth" so that no vitil information would be lost by interpolating botween measured points, then wider measurenent steps are adequate. Secondly, the formalism represented by Ëqs. (41)-(49) assumes that the two experiments have identical energy scales. The difficulty encountered wher. there are systematic differences in energy scales is deronstrated for the case of an isolated resonance in Fig. 4. Comparison at specific energies may be misleading even if the data sets exhibit consistent overal 1 normalization. Energy scale definition is crucial when comparing the resuits of measurements where the resolution functions $R_{1}$ and $R_{2}$ are similar and isolated resonances appear in the measured excitation functions. A well-knowr exampie is the neutron totai cross section for ${ }^{6} \mathrm{Li}$ in the vicinity of the $250-\mathrm{keV}$ resonance. 9 Measurements must be performed in increments which are small compared with the resolution of the experiment in order to locate the peak of the resonance and define the average cross section at that energy. Measurements near threshold must be conducted with similar care, otherwise, comparison of data sets may be misleading. Clearly, it is not meaningful to compare data sets with similar resolution unless both excitation functions have been measured in increments not exceeding their resolution widths $W_{1}$ and $W_{2}$.

When the resolution functions of two measurements are not equal, yet one cannot be said to be substantial broader than the other, it is generally impractical to seek a third function $R_{3}$ to be used for comparison of the results. Overall normalization of the data sets can be compared by averaging both sets with a resolution function $R_{4}$ which is much broader than either $R_{1}$ or $\mathrm{R}_{2}$ - Then, 
$\vec{\sigma}(E)=\int_{-\infty}^{\infty} R_{4}(\lambda-E) \sigma(\lambda) d \lambda=\int_{-\infty}^{\infty} \int_{-\infty}^{\infty} R_{4}(\lambda-E) \delta\left(\lambda^{\prime}-\lambda\right) \sigma\left(\lambda^{\prime}\right) d \lambda^{\prime} d \lambda$ $\approx \int_{-\infty}^{\infty} \int_{-\infty}^{\infty} R_{4}(\lambda-E) R_{1}\left(\lambda^{\prime}-\lambda\right) \sigma\left(\lambda^{\prime}\right) d \lambda^{\prime} d \lambda$

$$
\approx \int_{-\infty}^{\infty} \int_{-\infty}^{\infty} R_{4}(\lambda-E) R_{2}\left(\lambda^{\prime}-\lambda\right) \sigma\left(\lambda^{\prime}\right) d \lambda^{\prime} d \lambda
$$

and comparison is meaningful; however, all information on structural detail is lost and comparison is with regard to normalization only. Asain, both sets to be compared need to be complete in the sense that the excitation functions are recorded in increments not exceeding the measurement resolution widths.

The method of averaging two data sets with a very hroad resolution function is appoaling and practical because it avolds the necessity for exact knowledge of the resolution functions for the experimental measurements. The shape of the resolution function $R_{4}$ is not critical for this analysis, but it should be broad enough to encompass several resonances in the region of comparison (if the cross section is highly fluctuating) so as to minimize the possibility of distortion by details of the resonance structure (see Fig. 5). 


\section{CONCLUIING: REMARKS}

It has been indicated in this report that the parameters of an experiment establish definite relationships between the microscoplc cross section, the resolution and the measured cross section. Furthermore, it has been implied that investignters should mike a roisoniabe atcompt to model their experiment in sufficient detail during the analysis of their data so that these relationships can be determined and reported along with the cross sections. While this procedure is conceptually straightforward, considering the analytical power offered by modern computers, there are problems associated with the determination and control of the basic experimental parameters (e.g. targets may lose mass for various reasons or oxidize and broaden the resolution during the course of an experiment, etc.). To achieve the experimental control required to justify the extra effort entalled in the detailed analysis procedures described in this report, it is necessary for experimenters to impliment monitoring techniques which are often not included in typical experiments. For example, such measures will be essential in future activation experiments aimed at resolving integraldifferential discrepancles for several important dosimetry reactions. It has been demonstrated that the results of these measurements are quite sensitive to the energy scale. 10

Careful monitoring of the critical parameters of an experiment should enable the experimenter to establish realistic uncertainties for these parameters. Detailed analysis of the dnea will, in turn, establish the sensitivity of the mensured cross sections to these parameters and thereby permit a determination of the systumatic errors of the experiment. There Is strong evidence that serions systematic errors hove been overlooked in many experiments and the quoted errors are often predominanty statistical. 11 The inadequacy of quoted errors for experimental data currently hampers attempts to evaluate these data nonsubjectively or to examine sensitivity effects in nuclear data applications, 12,13

When planning experiments to measure cross sections with resonance structure, experimenters should select energy increments at least equal to their anticipated resolution. Comparison of isolated data points from various experiments is meaningless under these conditions. For data sets which do span a region of interest in some detail, comparison is possible provided both sets have very similar resolution, or if both sets are averaged with a resolution far broader than both experimental resolutions. In the latter case, normalization consistency alone is determined since the averaging process discards all structural information.

\section{ACKNONLEDGEMENTS}

The author is indebted to G. Winkler, J. W. Meadows, A. B. Smith, $P$, Guenther and $W$. Poenitz for the insight which they provided during many fruitful discussions on this subject. 


\section{REFERENCES}

1. "Neutron Cross Sections for Reactor Dosimetry, Vol. I-Review Papers." Froceedings of a consultants' meeting on integral cross-section measurements in standard neutron fields for reactor dosimetry organized by the International Atomic Energy Agency and held in Vienna, 15-19 November, 1976. Report IAEA-208, complled by M. Vlasov, Scientific Secretary (1978).

2. Donald L. Smith, "Remarks Concerning the Accurate Measurement of Differential Cross Sections for Threshold Reactions used in FastNeutron Dosimetry for Fission Reactors," Report ANL/NDM-23, Argonne National Laboratory (1976).

3. "Compilation of Requests for Nuclear Data," Report BNL-NCS-50632, compiled and edited by the National Nuclear Data Center for the ERDA Nuclear Data Committee, Brookhaven National Laboratory (1977).

4. A. B. Smith and J. F. Whalen, "Comments on the Energy-Averaged Total Neutron Cross Sections of Structural Materials," Report ANL/NDM-33, Argonne National laboratory (1977).

5. D. I. Smith, "A Spectrometer for the Investigation of Gamma Radiation Produced by Neutron-Induced Renctions," Report ANL/NDM-12, Argonne National Laboratory (1975).

6. C. Philis, R. llowerton and A. B. Smith, "Pltanium II: An Evaluated Nuclear Datia lile," Report ANL/NDM-28, Argonne National Laboratory (1977).

7. K. H. Beckurts and k. Wirtz, Neutron Physics, Springer-Verlag, Now York (1964).

8. Carl Eckart, Phys. Rev. 51, 735 (1937).

9. A B. Smith, P. Guenther, D. Havel and J. F. Whalen, "Note on the 250-keV Resonance in the Total Neutron Cross Section of ${ }^{6}$ LI," ANL/NDM-29, Argonne National Laboratory (1977).

10. Dona1d L. Smith, "Analysis of the Sensitivity of Spectrum-Average Cross Sections to Individual Characteristics of Differential Excitation Functions," ANL/NDM-30, Argonne National Laboratory (1977).

11. D. I. Garber and R. R. Kinsey, "Neutron Cross Sections, Vol. II, Curves," BNL-325, Third Edition, Brookhaven National Laboratory (1976).

12. F. G. Perey, "Least-Squares Dosimetry Unfolding: The Program STAY'SL", ORNL/TM-6062, Oak Ridge Nationa1 Laboratory (1977).

13. J. H. Marable, J. L. Lucius and C. R. Weisbin, "Compilation of Sensitivity Profiles for Several CSEWG Fast-Reactor Benchmarks," ORNL-5262, Oak Ridge National Laboratory (1977). 


\section{FIGURE CAPTIONS}

Fig. 1. Experimental values of the cross section for inelastic neutron scattering to the $0.847-\mathrm{MeV}$ state in ${ }^{56} \mathrm{Fe}$. (ANL Neg. No. 116-76-33).

Fig. 2, Typical energy resolution functions derived from the analysis of a gamma-ray experiment. Profiles are given for flux measurement with a fission chamber and for gamma-ray production measurements with a $\mathrm{Ge}\left(\mathrm{Li}_{i}\right)$ detector. Average neutron energies are indicated. The FWHM for these distributions are of the order of $100 \mathrm{keV}$; the distributions are different for flux measurements and the gamma-ray measurements because of the significant differences in the geometries. (ANL Neg. No. $116-79-74$ ).

Fig. 3A. The true cross section can be deduced readily from the measured results provided that the variation is gradual within the range of the resolution function.

(ANL Neg. No. 116-79-219).

3B. When the resolution function spans several resonance peaks, it is not possible to derive the true cross section from broad resolution data. (AM Neg. No, 116-79-219).

Fig. 4. The results of two measurements cannot be compared readily if their energy scales differ in an undetermined manner. (ANL Neg. No. 116-79-220 Rev. 1).

Fig. 5. In order to compare two sets of data with different resolutions $R_{1}$ and $R_{2}$, it is reasonable to average both sets with a resolution function $R_{4}$ which is much broader than either experimental function and broad enough to include many resonances in the microscopic cross section $\sigma$. (ANL Neg. No. 116-79-221). 


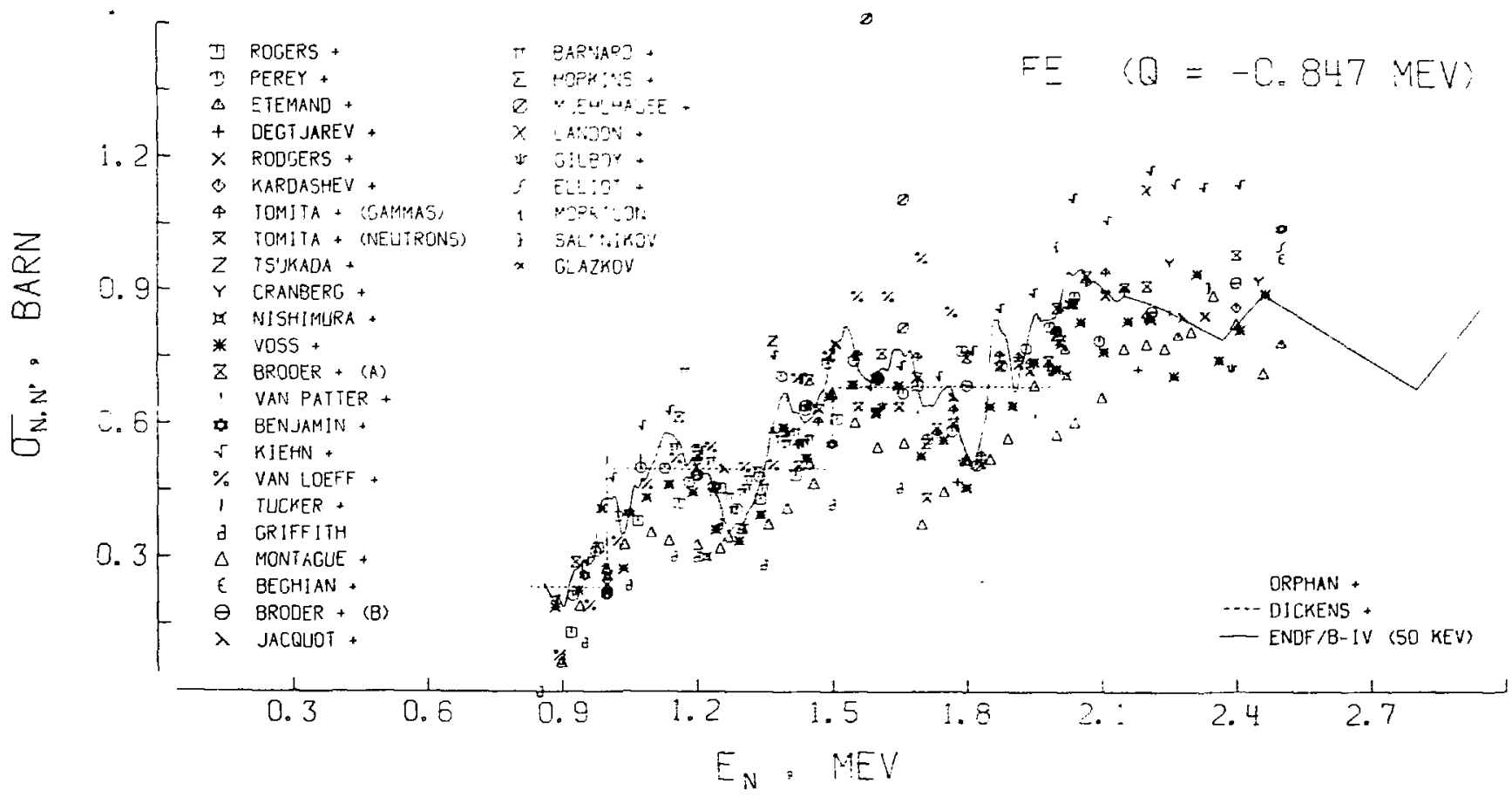




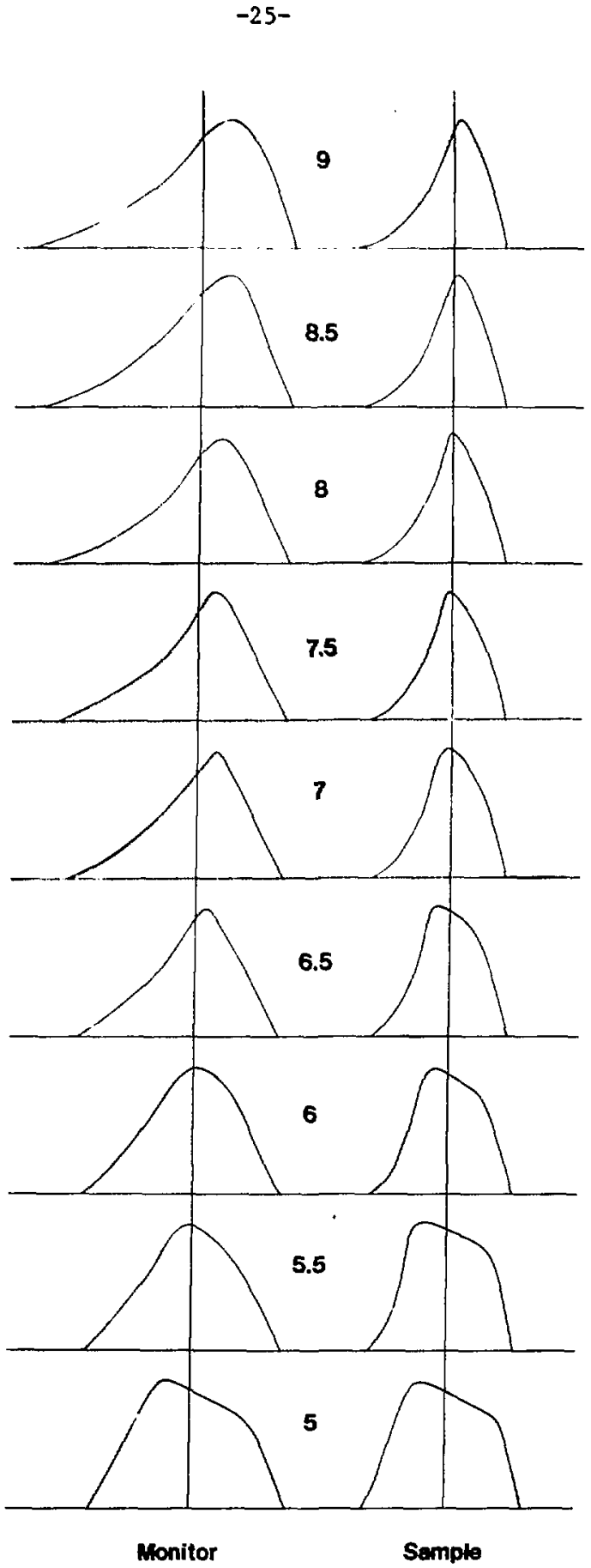

Eigure 2. 
A.
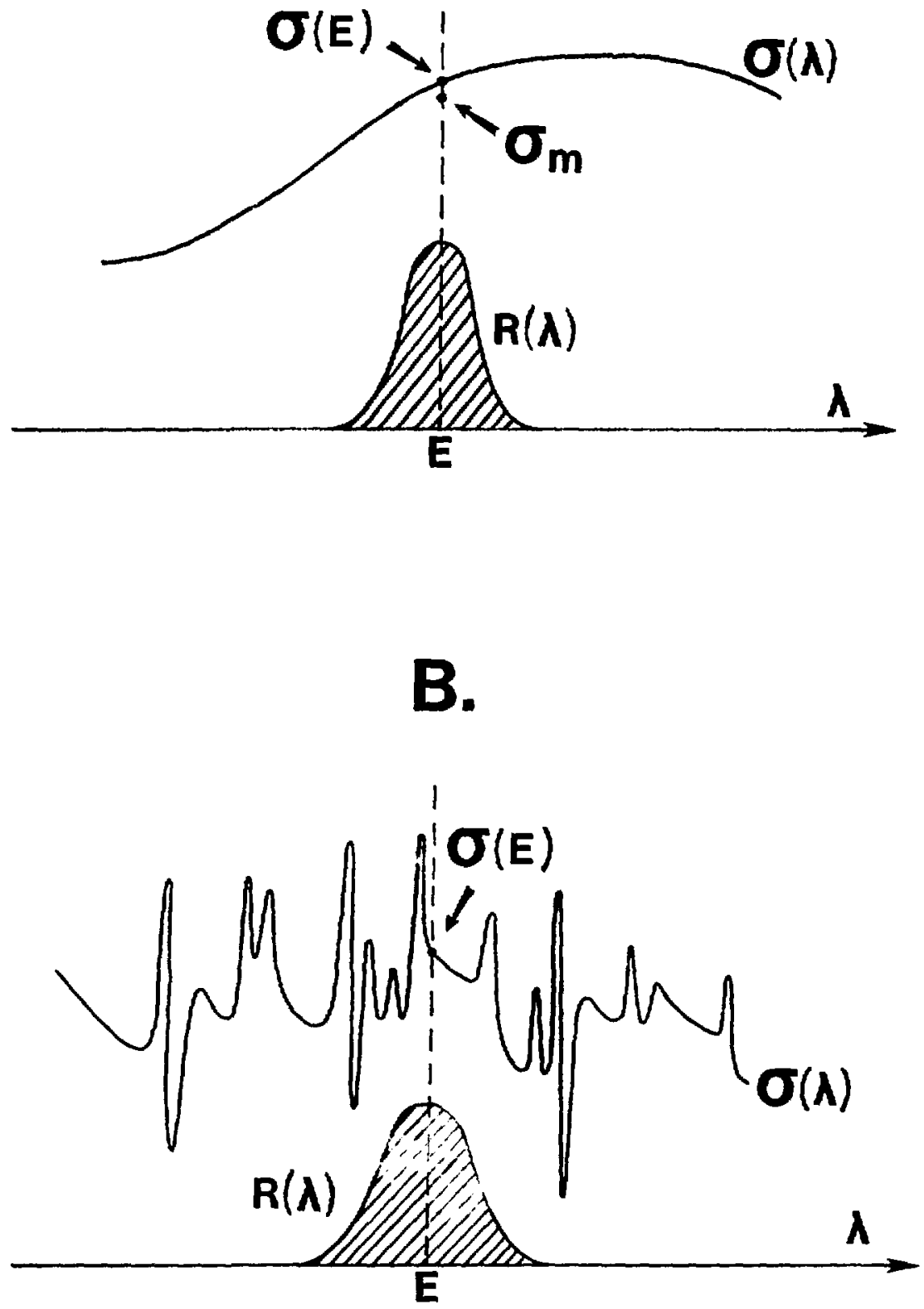

Figure 3. 


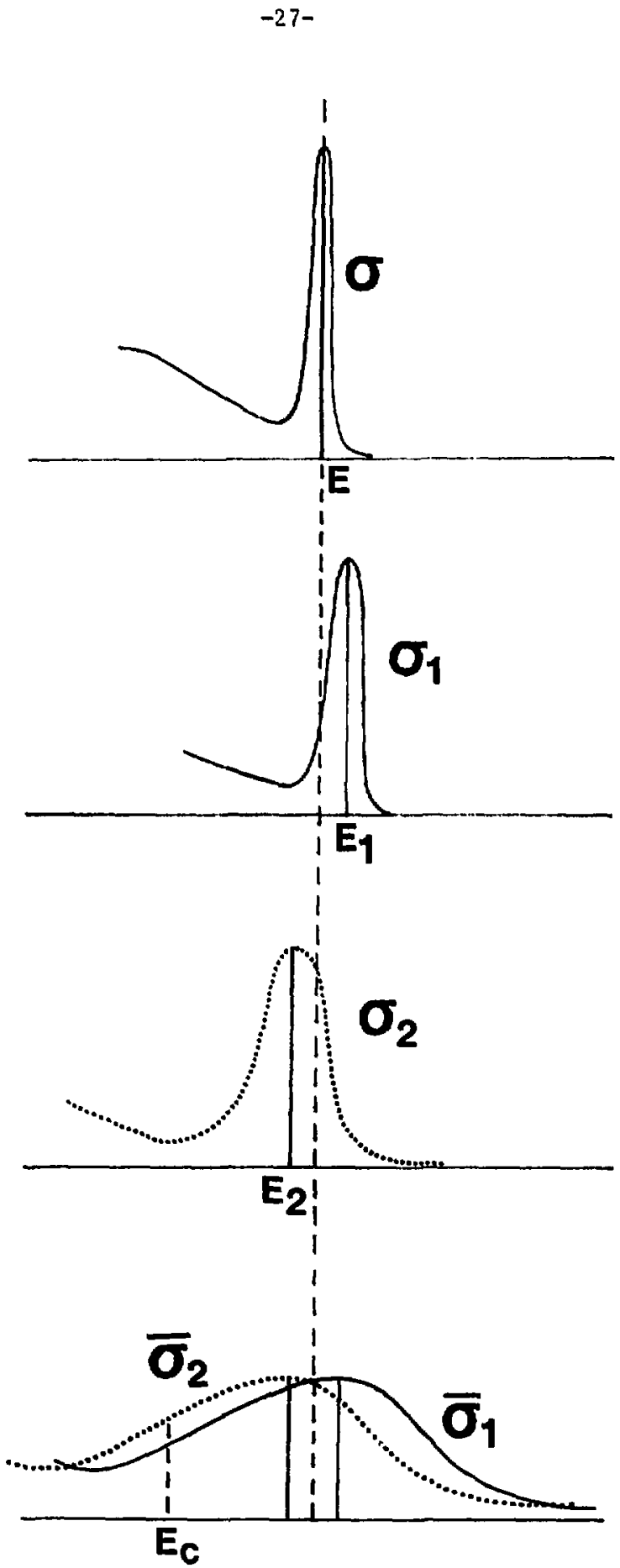

Figure 4. 
$-28-$
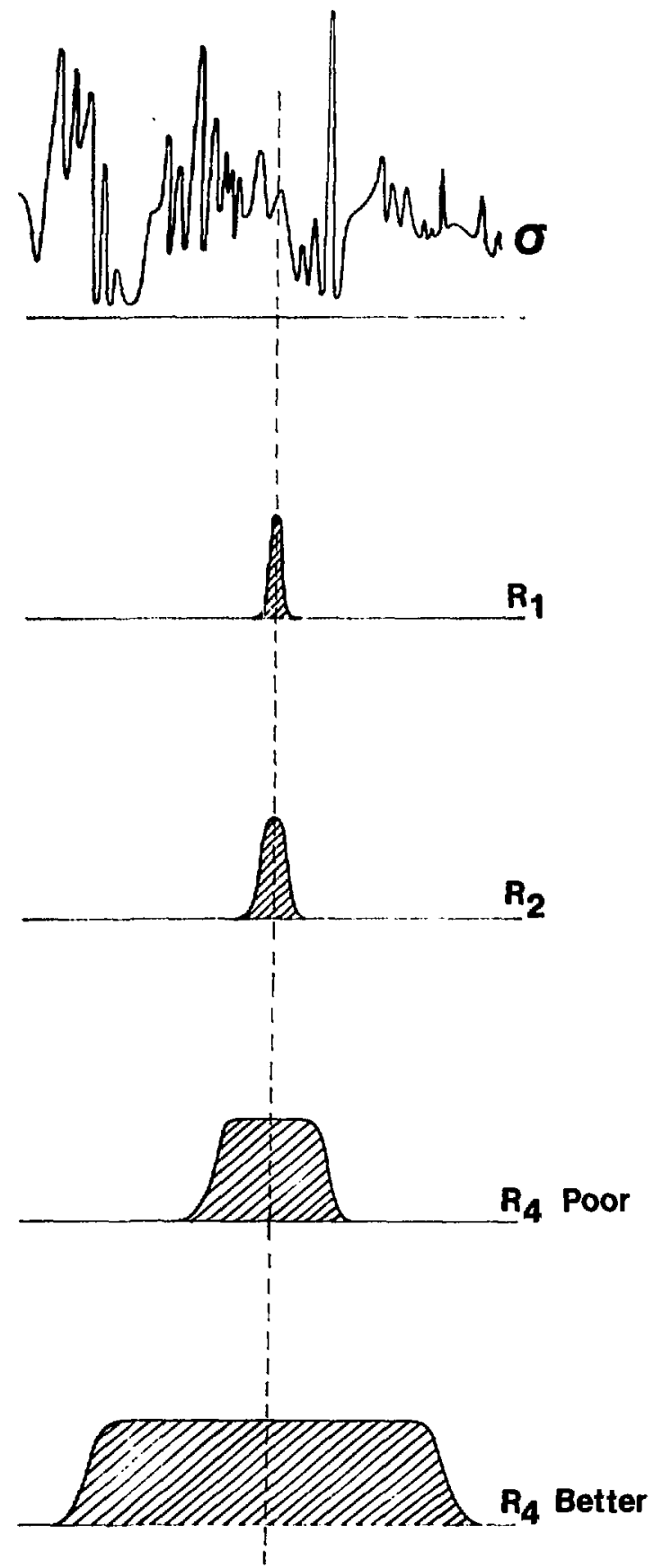

Figure 5. 